\title{
Dynamic coupling between a multistable defect pattern and flow in nematic liquid crystals confined in a porous medium
}

\author{
Takeaki Araki \\ Department of Physics, Kyoto University, Kyoto 606-8502, Japan
}

\begin{abstract}
When a nematic liquid crystal is confined in a porous medium with strong anchoring conditions, topological defects, called disclinations, are stably formed with numerous possible configurations. Since the energy barriers between them are large enough, the system shows multistability. Our lattice Boltzmann simulations demonstrate dynamic couplings between the multistable defect pattern and the flow in a regular porous matrix. At sufficiently low flow speed, the topological defects are pinned at the quiescent positions. As the flow speed is increased, the defects show cyclic motions and nonlinear rheological properties, which depend on whether or not they are topologically constrained in the porous networks. In addition, we discovered that the defect pattern can be controlled by controlling the flow. Thus, the flow path is recorded in the porous channels owing to the multistability of the defect patterns.
\end{abstract}

Topological defects are observed in various types of phases associated with spontaneous symmetry breaking such as crystals and ferromagnetic materials 11. In the early stage of the phase transitions, many defects are created, and their total amount then decreases with time to reduce the free energy [2. If frustrations are internally included or imposed by external fields, the defects are sometimes stabilized. On the other hand, re-organization of topological defects is often important under non-equilibrium conditions. For instance, pinning and depinning of quantized vortices in a type IIsuperconductor induce electrical resistance [3]. Motions of quantized vortices also lead to mutual frictional interactions in a flowing superfluid [2].

A nematic liquid crystal (NLC) is an ideal system for studying behaviors of topological defects because of its slow dynamics and large lengthscale [4 6]. When NLCs are confined in cavities [7, 8, microfluidic devices [9, 10, and porous media 11-17, the anchoring interaction between the director and the solid surface imposes frustrations on the elastic field. In a complex geometry, many topological defects in NLCs are thus sustained for a long time 6, 8, 18, 21. A NLC in a porous material exhibits slow glassy behavior and the resulting memory effects [15, 16, 19. In a previous paper, we studied the memory effect by Monte Carlo simulations, focusing on the role of the bicontinuity of the matrix [21. After cooling from an isotropic state, many disclination lines running through the channels of the porous network remain, with numerous possible trajectories. Because each trajectory is at one of the local energy minimum states and the energy barriers among them are often larger than the thermal energy, the system shows non-equilibrium behaviors similar to those in spin glasses. We found that the memorization is attributed to the re-configurations of the defect structure. The applications of an electric or magnetic field can bring the system into a new different state, and the new configuration remains even after the field is removed. Here, irreducible disclinations, which are topologically concatenated to the solid matrix, tend to enhance the memory effect.
In this Letter, we study a NLC flowing in a regular porous medium. In particular, we focus on dynamic couplings between the flow and the nonergodic disclination pattern. We hope our findings will facilitate research on microfluidics using liquid crystals [9, 10].

First, we prepare a pattern for a porous material by introducing a scalar variable $\phi$ in a cubic lattice according to Ref. 21]. $\phi$ varies smoothly in space. Then, we partition the cubic lattice into three portions by using $\phi: \mathcal{P}$ is the ensemble of lattice sites representing the solid material of the porous matrix; $\mathcal{N}$ denotes the nematic fraction and $\mathcal{S}$ is the ensemble of lattice sites at the interface. For the portions $\mathcal{N}$ and $\mathcal{S}$, we introduce a nematic tensorial order parameter $Q_{\alpha \beta}$ [22]. We employ the Landau-de Gennes free energy density 23,

$$
\begin{aligned}
& f_{\mathrm{LdG}}\left(Q_{\alpha \beta}\right)=\frac{\alpha_{\mathrm{F}}}{2} Q_{\alpha \beta} Q_{\beta \alpha}-\beta_{\mathrm{F}} Q_{\alpha \beta} Q_{\beta \mu} Q_{\mu \alpha} \\
& +\gamma_{\mathrm{F}}\left(Q_{\alpha \beta} Q_{\beta \alpha}\right)^{2}+\frac{L}{2}\left(\nabla_{\mu} Q_{\alpha \beta}\right)^{2}-\Delta \varepsilon E_{\alpha} E_{\beta} Q_{\alpha \beta},
\end{aligned}
$$

where $\alpha_{\mathrm{F}}, \beta_{\mathrm{F}}, \gamma_{\mathrm{F}}, L$ and $\Delta \varepsilon$ are phenomenological material constants. $\boldsymbol{E}$ is an external field. The repeated subscripts indicate summations over Cartesian indices. The free energy functional of the system is given by

$$
\mathcal{F}\left\{Q_{\alpha \beta}\right\}=\int_{\mathcal{N}+\mathcal{S}} d \boldsymbol{r} f_{\mathrm{LdG}}\left(Q_{\alpha \beta}\right)-w \int_{\mathcal{S}} d \boldsymbol{r} Q_{\alpha \beta} d_{\alpha} d_{\beta},(2)
$$

where $\boldsymbol{d}$ is the normal vector of the porous matrix, $\boldsymbol{d}=\nabla \phi /|\nabla \phi|$. The second term represents the molecular anchoring interaction between the surface of the porous matrix and the nematic orientational order, and $w$ is its material constant. We assume $w>0$, i.e., that homeotropic anchoring is preferred.

The hydrodynamic flow $\boldsymbol{u}$ is determined by a momentum equation [23],

$$
\rho\left(\frac{\partial}{\partial t}+u_{\mu} \nabla_{\mu}\right) u_{\alpha}=\nabla_{\beta}\left(-p \delta_{\alpha \beta}+\sigma_{\alpha \beta}^{\mathrm{d}}+\sigma_{\alpha \beta}^{\prime}\right),
$$

where $\rho$ is the material density and $p$ is the pressure. Here, $\sigma_{\alpha \beta}^{\mathrm{d}}$ is the distortion stress tensor, which is given 
by

$$
\sigma_{\alpha \beta}^{\mathrm{d}}=-\frac{\partial f_{\mathrm{LdG}}}{\partial\left(\nabla_{\alpha} Q_{\mu \nu}\right)}\left(\nabla_{\beta} Q_{\mu \nu}\right)
$$

Further, $\sigma_{\alpha \beta}^{\prime}$ is the viscous stress tensor, which is given by

$$
\begin{aligned}
\sigma_{\alpha \beta}^{\prime}= & \beta_{1} Q_{\alpha \beta} Q_{\mu \nu} A_{\mu \nu}+\beta_{4} A_{\alpha \beta}+\beta_{5} Q_{\alpha \mu} A_{\mu \beta}+\beta_{6} Q_{\beta \mu} A_{\mu \alpha} \\
& +\frac{1}{2} \mu_{2} N_{\alpha \beta}-\mu_{1} Q_{\alpha \mu} N_{\mu \beta}+\mu_{1} Q_{\beta \mu} N_{\mu \alpha}
\end{aligned}
$$

where $A_{\alpha \beta}=\left(\nabla_{\alpha} u_{\beta}+\nabla_{\beta} u_{\alpha}\right) / 2$ is the symmetric velocity gradient tensor, and $N_{\alpha \beta}$ is the time rate of change of $Q_{\alpha \beta}$ with respect to the background flow. The latter is defined as

$$
N_{\alpha \beta}=\frac{\partial}{\partial t} Q_{\alpha \beta}+u_{\mu} \nabla_{\mu} Q_{\alpha \beta}+\omega_{\alpha \mu} Q_{\mu \beta}-Q_{\alpha \mu} \omega_{\mu \beta},
$$

where $\omega_{\alpha \beta}=\left(\nabla_{\alpha} u_{\beta}-\nabla_{\beta} u_{\alpha}\right) / 2$ is the asymmetric velocity gradient. In Eq. (5), $\beta_{i}(i=1,4,5,6)$ and $\mu_{i}(i=1,2)$ are phenomenological parameters having the dimension of viscosity. The time development of the nematic order parameter is given by

$$
N_{\alpha \beta}=-\frac{1}{\mu_{1}} \frac{\delta \mathcal{F}}{\delta Q_{\alpha \beta}}-\frac{\mu_{2}}{2 \mu_{1}} A_{\alpha \beta} .
$$

The above nemato-hydrodynamic equations are solved with non-slip boundary conditions at the surfaces of the porous matrix.

Lattice Boltzmann simulation is one of the most efficient numerical methods of investigating flow behaviors in a complex geometry [24]. The bounce-back method realizes non-slip boundary conditions on the velocity fields at the surfaces of the porous matrix [24]. To our knowledge, two lattice Boltzmann algorithms for simulating nemato-hydrodynamics have been proposed [2528. In this study, we adopt a numerical scheme developed by Care and coworkers. Here, we omit the details and follow their notations (see Refs. [26, 27]). We performed three-dimensional simulations with four speeds and coordination number 27 (D3Q27). The weight factors of the four speeds $t_{i}$ are given by $t_{i}=8 / 27$ for $\boldsymbol{c}_{i}=(0,0,0), t_{i}=2 / 27$ for $\boldsymbol{c}_{i}=( \pm 1,0,0), t_{i}=1 / 54$ for $\boldsymbol{c}_{i}=( \pm 1, \pm 1,0)$ and $t_{i}=1 / 216$ for $\boldsymbol{c}_{i}=( \pm 1, \pm 1, \pm 1)$ with appropriate permutations. Here, $\boldsymbol{c}_{i}$ is the nondimensional velocity vector.

In our simulations, we impose an external force density $f$ to flow the NLC. Then, we modify Eq. (31) in Ref. [26] to

$$
\begin{aligned}
& \sum_{j} M_{i \alpha \beta j \mu \nu} g_{j \mu \nu}^{(\mathrm{neq})}=-\frac{g_{i \alpha \beta}^{(\mathrm{neq})}}{\tau_{i}}+\delta m_{i \alpha \beta}+\delta p_{i \alpha \beta} \\
& +\sum_{i} \rho t_{i}\left\{f_{\mu}\left(c_{i \mu}-u_{\mu}\right)+f_{\mu} u_{\nu} c_{i \mu} c_{i \nu}\right\} \delta_{\alpha \beta},
\end{aligned}
$$

where $\sum_{j}$ represents summation over 27 velocity vectors $\boldsymbol{c}_{j}$. We employ $\alpha_{\mathrm{F}}=-0.133, \beta_{\mathrm{F}}=2.133, \gamma_{\mathrm{F}}=1.6, w=$ $0.1, L=0.1, \Delta \varepsilon=1.0, \mu_{1}=3.0, \mu_{2}=-1.0, \tau_{0}=1.1$, $\eta_{0}=0.3$, and $\eta_{2}=0.5$. This set of parameters gives a correlation length $\xi=0.87 \Delta$, an anchoring penetration length $d_{\mathrm{a}}=\Delta$, and a bulk nematic order $Q_{\mathrm{b}}=0.37$, where $\Delta$ is the unit cell length. The resulting viscosity ratio of this anisotropic liquid is $\eta_{\perp} / \eta_{\|} \cong 1$.1. Here $\eta_{\|}$ and $\eta_{\perp}$ are the effective viscosities when the director is parallel to the flow and parallel to the velocity gradient, respectively. We adopt a bicontinuous pattern with cubic symmetry, which we have studied and is denoted as BC in Ref. 21. The size of the unit cell of the bicontinuous cubic is $L=32 \Delta$. We use a simulation box of $64^{3}$ with periodic boundary conditions.

In NLCs, the flow speed is characterized well by the Ericksen number $E r$, which is the ratio of the viscous and the elastic forces. It is given by $E r=|\boldsymbol{f}| \ell^{3} / K$, where $K$ is the Frank elastic modulus expressed as $K=9 L Q_{\mathrm{b}}^{2} / 2$ in a one-constant approximation, and $\ell$ is the characteristic length of the distortion. Because it is difficult to determine $\ell$ quantitatively in a complex geometry, we assume that $\ell$ is of the order of the radius of the narrowest channels, namely, $\ell=L / 4$.

We quenched the system from an isotropic state to a nematic state without any external field $\boldsymbol{E}$ or bulk force $\boldsymbol{f}$. Because of the anchoring effect, the director was strongly deformed, therefore, disclination lines remained even after long duration annealing. Figure 1(a) shows one of the stable defect configurations, where the defect positions are represented by the isosurfaces of the elastic energy density $f_{\mathrm{el}}=L\left(\nabla_{\mu} Q_{\alpha \beta}\right)^{2} / 2$. Here, the remaining disclinations run through the channels rather randomly. They are closed without ends, and some are concatenated to the solid matrix. Then, we applied a strong external field pulse along the $z$-axis $\left(E_{z}=0.1\right)$. After the field was removed, two types of disclination loops formed in the $x-y$ planes, as shown in Fig. 1(b). Half of the remaining disclinations (red loops in Fig. 1(b)) are localized at the narrowest sections of the channels. The line defects locally have the topological charge $s=-1 / 2$, so the loops are reducible continuously into hyperbolic point defects with $s=-1$. On the other hand, the other half of the loops (blue loops in Fig. 1 (b)) consists of disclinations, which locally have the topological charge $s=1 / 2$. However, they are irreducible to point defects, because the solid necks of the $\mathrm{BC}$ penetrate the loops. Hereafter, the former and latter defect groups are referred to as type-I and II, respectively. This structure is the same as that obtained in the Monte Carlo simulations [21]. It also appears that this configuration is probably one of the global energy minimum states, which are also degenerated along the $x$ and $y$-axes. We employ this configuration as an initial state.

First, we impose force $f_{z}$ to flow the NLC toward the $z$-direction. In NLCs, defect motion is caused by not only hydrodynamic convection, but also rotations of the director field. If the imposed force is weak enough, both types of defects show only small displacements, which are determined by the balance between hydrodynamic convection and director rotation (not shown here). As the flow 
speed is increased, the director rotation can no longer compensate for the hydrodynamic convection, so the defect pattern becomes unstable against the flow. Figure 2 shows the temporal changes in (a) the defect pattern and (b) the cross section of $Q_{z x}$ at an $x-z$ plane that cuts the centers of the necks of the BC. The applied force strength is $f_{z}=0.006$, which corresponds to $E r \cong 50$. Under this force, the type-I defects start to exhibit cyclic behavior. As shown in Fig. 2(b), the director field is gradually distorted with time. When the elastic distortion energy becomes comparable to the anchoring energy, the anchoring at the downstream side of the porous surface is transiently broken (see $t=270$ in Fig. 2(b)). When the anchoring breaks, new defects are born from the surface. Then, the new defects and the old type-I loops fuse into new type-I disclination loops. During this cycle, the type-II loops keep to be at the quiescent positions. With a further increase in the flow speed, the type-II loops also become unstable. Figure 2(c) shows snapshots of the defect pattern at $f_{z}=0.013$. Because they are concatenated to the channels, they cannot move without a topological transformation. The type-II defects repeat another cycle, in which they are deformed, scissored and reconnected sequentially.

In Fig. 3(a), we plot the temporal changes in the averaged orientational order along the $z$-axis $\left\langle Q_{z z}\right\rangle$. When the force is weak $\left(f_{z} \lesssim 0.004\right)$, the orientational order is almost fixed at the same value as that at rest. Subject to an intermediate force $\left(0.006 \lesssim f_{z} \lesssim 0.008\right)$, the orientational order shows an oscillation mode, which corresponds to the repeated cycle of the type-I defects shown in Figs. 2(a) and (b). For strong force $\left(f_{z} \gtrsim 0.010\right)$, two oscillation modes are observed in $\left\langle Q_{z z}\right\rangle$. The mode of the shorter period is the same as that for $f_{z}=0.006$; on the other hand, that of the longer period corresponds to the cycle for the type-II defects shown in Fig. 2(c). The frequencies of the two oscillation modes are shown in Fig. 3(b). Both frequencies decreased with decreasing $E r$. It is likely that they show critical behaviors as $\omega \propto\left|E r-E r_{\mathrm{c}}\right|^{k}$ with $k=1.0$. The critical values of $E r$ are estimated to be $E r_{\mathrm{c}}^{\mathrm{I}} \cong 26$ for the type-I defects and $E r_{\mathrm{c}}^{\mathrm{II}} \cong 64$ for type-II defects by extrapolating straight lines. However, the true critical points are not accessible because of our simulation's constraints. Therefore, we cannot yet conclude whether these bifurcations are continuous or not, and determine the exponent $k$.

Figure 3(c) shows the temporal changes in the flow speeds $\left\langle u_{z}\right\rangle$ averaged inside the porous matrix. Above the critical strength for the cyclic defect behavior, the flow speed exhibits oscillation, the period of which coincides with that of $\left\langle Q_{z z}\right\rangle$. In Fig. 3(d), we plot the apparent viscosity $\eta_{z}$, which is obtained by averaging it over $1000 \leq t \leq 2000$. Here, the viscosity is normalized by that of an isotropic liquid $\eta_{\text {iso }}$, which is determined by the same simulation method. Below the critical force, the apparent viscosity decreases marginally with $E r$. That is, the NLC exhibits a shear-thinning rheology. At the two critical values of $E r$ for the onset of the oscillations, small kinks in the apparent viscosity are observed, indicating that the defect pattern influences the flow properties.

Next, we impose an external force toward the $x$ direction. Under a weak force, all the defects are pinned at their original positions, showing marginal elongations along the flow (not shown here). Above a certain force, we found that the defect pattern switched to an orientation along the flow direction. The switching process of the defect pattern under $f_{x}=0.005$, which corresponds to $E r \cong 42$, is shown in Fig. 4. Once the orientation is switched, the new defect pattern is sustained owing to its multistability even after the flow is stopped. The apparent viscosity for the $x$-oriented flow, $\eta_{x}$, is also plotted in Fig. 3(d). Below the critical Ericksen number for switching, the apparent viscosity along the $x$-axis is larger than $\eta_{z}$. At the critical Ericksen number $\left(E r_{\mathrm{c} \perp} \cong 25\right), \eta_{x}$ decreases abruptly to $\eta_{z}$. After switching, the system exhibits the same oscillations as those for the parallel flow in Fig. 2.

Note that the oscillation frequencies and the corresponding Ericksen number depend on the anchoring strength, because the anchoring breaking plays a crucial role in the cyclic behaviors of the topological defects. Thus, the values displayed are not universal for any anchoring strength and pore size. Moreover, qualitatively distinct behaviors may be observed in extreme cases. For instance, defects may be transported unboundedly without the cyclic motions if the anchoring strength is infinitely large, although our simulations cannot access this regime. Because the Ericksen number does not factor in the anchoring effect, it cannot describe all the flow properties. If the morphology of the porous matrix is the same, the director pattern is characterized by a nondimensional parameter $W=w L / K$ in the absence of flow. Two dimensional mapping of the non-equilibrium behaviors onto a $W-E r$ plane will help to understand the flow properties of NLCs in confined systems. We need to study them more quantitatively.

We studied the flow behaviors of a NLC in a porous medium by lattice Boltzmann simulations. In the case of strong anchoring, the pattern of the director field can adopt many (meta-)stable configurations. We found that this multistability leads to peculiar flow properties of the confined NLCs. The dynamic behaviors of topological defects under flow depend on whether they are topologically concatenated to the matrix.

We also found that the apparent viscosity depends on the mutual direction of the flow; i.e., the NLC flows easily along the orientation of the averaged director field. Imposing an intermediate flow along an incommensurate direction switches the global director field to the new direction. Upon this switching, the apparent viscosity decreases abruptly to that for the commensurate flow. Because this defect pattern is maintained even after the flow is stopped, we can say the flow path can be recorded in the porous network. As reported in a previous paper, the emergence of topological defects enhance the efficiency of memorization 21. We also expect that the flow path in 
a porous matrix can be dynamically selected by changing the multistable defect patterns. In our simulations, the difference between the apparent viscosities of the parallel and perpendicular flows is not large compared to that in Ref. 26]. In other words, our system is more isotropic in viscosity. This small viscosity difference is attributed to the employed parameters, which are restricted in our present scheme. When a NLC having a large viscosity anisotropy is used, the difference in the apparent viscosity will be more remarkable.

We employed a bicontinuous matrix with cubic symmetry as an example. Because the configuration of topological defects depends on the morphology of the network, our results cannot be directly applied to any matrices. However, we consider that dynamic coupling between the multiple stabilities and the flow will be found ubiquitously for NLCs confined in any porous network. We also hope our results will improve the understandings of the flow properties of NLCs in porous media and microfluidic channels.

We acknowledge valuable discussions with H. Tanaka, T. Bellini, F. Serra, M. Buscaglia and A. Sengupta. This work was supported by the JSPS Core-to-Core Program
"International research network for non-equilibrium dynamics of soft matter" and KAKENHI. The computational work was performed using the facilities at the $\mathrm{Su}$ percomputer Center, Institute for Solid State Physics, University of Tokyo.

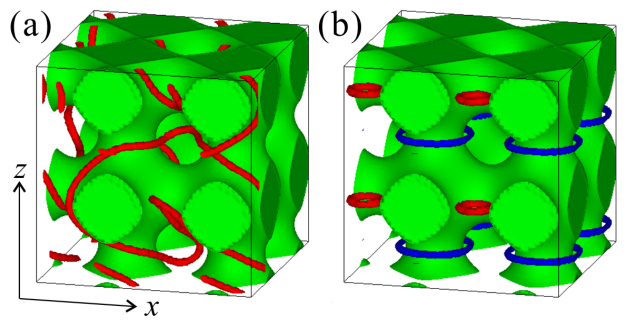

FIG. 1. (a) Snapshot of disclination lines of nematic liquid crystals in a porous medium after zero field cooling from an isotropic state. Solid green objects represent the porous matrix, and red curves are the remaining disclinations. (b) Defect pattern remaining after an external field pulse is applied along the $z$-axis. Red and blue curves represent reducible (type-I) and irreducible (type-II) disclinations, respectively.
[1] P. M. Chaikin and T. C. Lubensky, Principles of Condensed Matter Physics (Cambridge University Press, Cambridge, 1995).

[2] A. Onuki, Phase Transition Dynamics (Cambridge University Press, Cambridge, 2002).

[3] D. S. Fisher, M. P. A. Fisher, and D. A. Huse, Phys. Rev. B 43, 130 (1991).

[4] I. Chuang, N. Durrer, R. Turok, and B. Yurke, Science 251, 1336 (1991).

[5] G. P. Crawford and S. Žumer, Liquid Crystals in Complex Geometries Formed by Polymer and Porous Networks (Taylor and Francis, London, 1996).

[6] I. Muševič and S. Žumer, Nature Materials 10, 266 (2011).

[7] Z. Bradač, S. Kralj, and S. Žumer, Phys. Rev. E 58, 7447 (1998).

[8] F. Serra, K. C. Vishnubhatla, M. Buscaglia, R. Cerbino, R. Osellame, G. Cerullo, and T. Bellini, Soft Matter 7, 10485 (2011).

[9] A. Sengupta, U. Tkalec, and C. Bahr, Soft Matter 7, 6452 (2011).

[10] A. Sengupta et al., Microfluid Nanofluid, DOI:10.1007/s10404-012-1014-7 (2012).

[11] G. S. Iannacchione, G. P. Crawford, S. Žumer, J. W. Doane, and D. Finotello, Phys. Rev. Lett. 71, 2595 (1993).

[12] X.-l. Wu, W. I. Goldburg, M. X. Liu, and J. Z. Xue, Phys. Rev. Lett. 69, 470 (1992).

[13] T. Bellini, C. Chiccoli, P. Pasini, and C. Zannoni, Phys. Rev. E 54, 2647 (1996).

[14] T. Bellini, M. Buscaglia, C. Chiccoli, F. Mantegazza, P.
Pasini, and C. Zannoni, Phys. Rev. Lett. 85, 1008 (2000).

[15] T. Bellini, M. Buscaglia, C. Chiccoli, F. Mantegazza, P. Pasini, and C. Zannoni, Phys. Rev. Lett. 88, 245506 (2002).

[16] M. Buscaglia, T. Bellini, C. Chiccoli, F. Mantegazza, P. Pasini, M. Rotunno, and C. Zannoni, Phys. Rev. E 74, 011706 (2006).

[17] S. Gruener and P. Huber, J. Phys.: Condens. Matter 23, 184109 (2011).

[18] I. Muševič and M. Skarabot, Soft Matter 4, 195 (2008).

[19] M. Rotunno, M. Buscaglia, C. Chiccoli, F. Mantegazza, P. Pasini, T. Bellini, and C. Zannoni, Phys. Rev. Lett. 94, 097802 (2005).

[20] T. Araki and H. Tanaka, Phys. Rev. Lett. 97, 127801 (2006).

[21] T. Araki, M. Buscaglia, T. Bellini, and H. Tanaka, Nature Materials 10, 303 (2011).

[22] P. G. de Gennes and J. Prost, The Physics of Liquid Crystals (2nd Ed.) (Oxford University Press, Oxford, 1993).

[23] T. Qian and P. Sheng, Phys. Rev. E 58, 7475 (1998).

[24] S. Succi, The Lattice Boltzmann Equation for Fluid Dynamics and Beyond (Oxford University Press, Oxford, 2001).

[25] C. Denniston, E. Orlandini, and J. M. Yeomans, Phys. Rev. E 63, 056702 (2001).

[26] C. M. Care, I. Halliday, and K. Good, J. Phys.: Condens. Matter 12, L665 (2000).

[27] C. M. Care, I. Halliday, K. Good, and S. V. Lishchuk, Phys. Rev. E 67, 061703 (2003).

[28] T. J. Spencer and C. M. Care, Phys. Rev. E 74, 061708 (2006). 
(a)
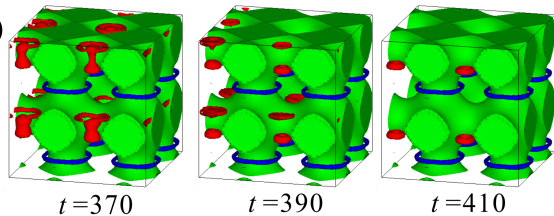

(b)
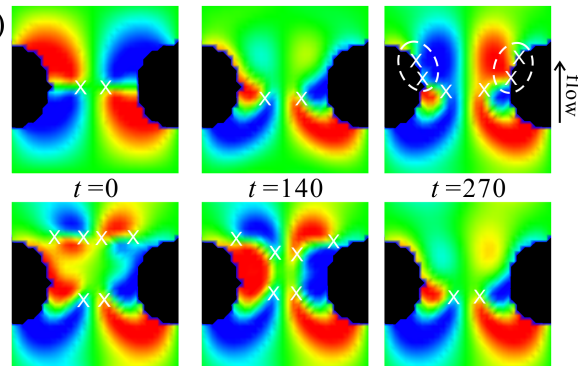

$t=330$

$t=140$

$t=270$

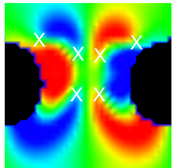

$t=350$

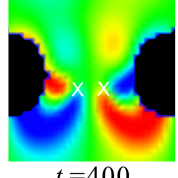

$t=400$

(c)
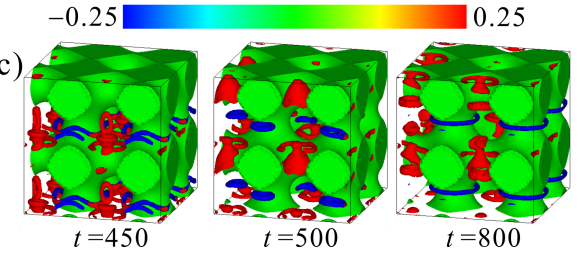

FIG. 2. Time evolutions of (a) the defect pattern and (b) $Q_{z x}$ at an $x-z$ plane under $f_{z}=0.006$. In (b), the black areas are the solid matrix, and white crosses indicate the defect positions. At $t=270$, the anchoring condition is broken. (c) Time evolution of the defect pattern under $f_{z}=0.013$. The type-II (blue) defects also exhibit cyclic behavior.
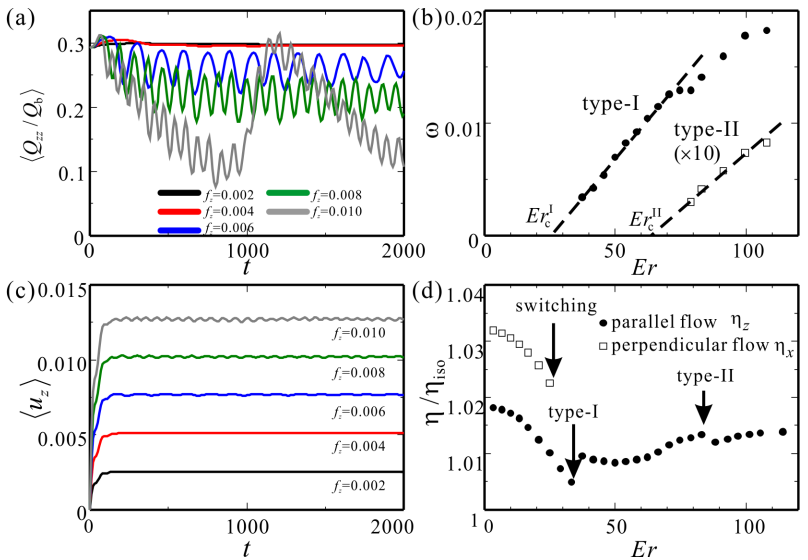

FIG. 3. (a) Temporal changes in the averaged remnant order $\left\langle Q_{z z}\right\rangle$. (b) Frequencies of the cyclic motions of the type-I and II defects as a function of the Ericksen number. Broken lines are provided for better visualization. (c) Temporal changes in the flow speed $\left\langle u_{z}\right\rangle$ of the liquid crystal in the porous matrix. The force density is increased from $f_{z}=0.002$ to 0.01 . (d) Effective viscosities for the parallel and perpendicular flows plotted with respect to $E r$. The viscosity is normalized by that of an isotropic liquid $\eta_{\text {iso. }}$. At the arrows, the defect patterns are re-organized. 


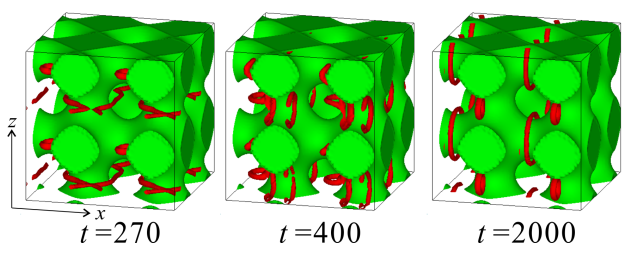

FIG. 4. Switching behaviors of topological defects under a perpendicular flow. A force $f_{x}=0.005$ is imposed for the defect pattern aligned along the $z$-axis. 